same reason opinions, whether diagnoses or problem definitions, would have to become more disciplined than they often are, while the judgmental statements we sometimes see in records would have to be omitted. Fisher et al quote the distress of a patient finding something that was "untrue, and a long time ago."

There are operational problems, but these should not be insurmountable. Assertions that patients would lose their records have to be set against the average non-availability of records at outpatient encounters and surgery attendances. Patients must be given time to read their records.

Perhaps the most cogent reason for at least delaying the introduction of sharing is the quality of the records themselves. Fisher et al report that only $44 \%$ of patients could read the general practitioner's writing and only one fifth said they could understand the notes. With a few honourable exceptions medical records (hospital or general practice) are a disgrace, and to share them with the patients would be to expose the extent to which the science based technology of medicine depends on an information system that has neither been rethought nor even taught in a disciplined way for $\lambda$ several practice lifetimes. Perhaps sharing records would $\mathbb{\infty}$ inculcate much needed discipline in the profession's record keeping.

Professor of General Practice,

DaVid METCALFE

University of Manchester,

Rusholme Health Centre,

Manchester M14 5NP

\title{
The case against showing patients their records
}

Nothing, so far as I can see, is to be gained by patients having access to their records, and a little may be lost. All doctors have a responsibility to explain fully to patients their diagnoses and management, and giving patients full access to what inevitably will be confusing and possibly frightening material may well hinder rather than enhance communication. Furthermore, it might create extra work and bureaucracy in a health service that is already struggling to cope with its heavy load.

If patients have full access to their records and to letters between consultants and general practitioners then, I believe, the nature of the information contained will change. Doctors will be more circumspect in what they say, which will benefit neither the patients nor the communication between doctors. Tentative diagnoses and plans will be excluded, as may sensitive (and yet possibly vital) information on, for instance, sexual or financial problems. Frank and often very useful descriptions of patient characteristics will be watered down, and the "thinking out loud" that is an important part of communication between doctors will be restricted to conversations and telephone calls_-and so eventually forgotten. Operation notes will be censored to leave out descriptions of the parts of the operation that did not go smoothly, because all that the patient wants to know is that by the end everything was well.

Although this issue of the journal contains reports on how patients in general practice have been glad to see their records (pp 595, 596, and 603), we must remember that inpatient hospital records are usually much fuller than general practice records. In addition, many more people write in hospital records. I imagine that if I were to have to explain to every patient the meaning of his having a slightly raised serum potassium value nine years ago then it might take up a lot of time that I could spend more usefully. But if I also have to explain what it was that an occupational therapist meant when she wrote some abstruse paragraph I will become very exasperated. And what about medical students writing in hospital notes? One might have got completely the wrong end of the stick and written down some fanciful but terrifying diagnosis. How will I explain that he was only a medical student and had little knowledge of surgery? Will the patient believe me? Will we have to stop medical students writing in records? That would be a loss.

I am worried not only by time that might be wasted in often irrelevant explanations, and by the alarm that might be created unnecessarily, but also by the work that will have to be done before opening up records to patients. The tradition has always been that records are not shown to patients, and doctors and others have written them accordingly. Many of those who will have contributed to the records will have moved on, retired, or died. If we are to show patients their records then we will have to sit down first and remove those parts of the records that might be misunderstood or create alarm. This will take time, and who is to do it? It will have to be a doctor and preferably the consultant in charge of the patient. He or she will have better ways of spending time, one of which might be explaining to patients what is happening to them-not in the obscure argot of medical records but in straightforward English that anybody can understand.

Another difficulty is that patients vary so much. The proponents of full disclosure of medical records have in mind, I suspect, middle aged, middle income, middle class patients who subscribe to Which? But what about children, mentally handicapped patients, and those who are profoundly disturbed or demented? Dr Dora Black, a child psychiatrist, has described well the anger she felt when letters she had written about a 3 year old child were handed on to the father's solicitor without her permission-through a general practitioner who gave his patients full access to their records. ${ }^{1}$ In this case the child was clearly not helped. Most consultants, I am sure, would want to know whether or not their comments to general practitioners were being passed on directly to patients. I certainly would.

This case brings me to my final worry about giving patients full access to records: difficulties may arise-as in Dr Black's case-because people other than the patient see the records. We will have our work cut out to censor records so they do not cause distress without having to try to take account of every single person who might have a chance to pass his eye over the record. I'm not passionately against patients seeing their records, and I do recognise that people have some sort of right to see any record kept about them, but I cannot see that patients will benefit from full access-and doctors will surely lose.

Consultant Surgeon,

Alexander P Ross

Royal Hampshire County Hospital,

Winchester SO22 5DG

1 Black D. Personal view. Br Med f 1985;291:1718 . 